\title{
Lung ultrasound may improve COVID-19 safety protocols
}

\author{
Szymon Skoczyński ${ }^{1}$, Natalia Buda ${ }^{2}$, Konrad Mendrala ${ }^{3}$, Tomasz Górecki ${ }^{4}$, Ewa Kucewicz-Czech ${ }^{3}$, \\ Lukasz Krzych ${ }^{3}$, Tomasz Koszutski ${ }^{5}$, Tomasz Darocha ${ }^{3}$
}

${ }^{1}$ Department of Pneumonology, Faculty of Medical Sciences in Katowice, Medical University of Silesia, Katowice, Poland; ${ }^{2}$ Internal Medicine, Connective Tissue Diseases and Geriatrics Department, Medical University of Gdańsk, Gdańsk, Poland; ${ }^{3}$ Department of Anaesthesiology and Intensive Care, Medical University of Silesia, Katowice, Poland; ${ }^{4}$ Department of Medical Education of the Jagiellonian University Medical College, Kraków, Poland; ${ }^{5}$ Department of Pediatric Surgery and Pediatric Urology, Upper Silesian Child Health Centre, School of Medicine in Katowice, Medical University of Silesia in Katowice, Katowice, Poland

Correspondence to: Szymon Skoczyński, MD, PhD. Department of Pneumonology, Faculty of Medical Sciences in Katowice, Medical University of Silesia, Medykow 14, PL-40752 Katowice, Poland. Email: simon.mds@poczta.fm.

Submitted Feb 19, 2021. Accepted for publication Apr 16, 2021.

doi: $10.21037 /$ jtd-21-295

View this article at: http://dx.doi.org/10.21037/jtd-21-295

\section{Introduction}

Since the first confirmed infection of a novel coronavirus labeled SARS-CoV2 the high number of COVID-19 associated acute respiratory distress syndrome (ARDS) and risk of virus transmission among health care providers are one of the problems of greatest concern. Even with the increasing number of COVID-19 vaccinations, accurate assessment and prudent safety regime should be prioritized. Also, since numerous cases in which a patient, who was not considered initially as infected with SARS-CoV2, was finally diagnosed as a COVID-19 case, the need of additional safety protocol is of the utmost importance. Every patient with acute respiratory infection with fever, cough, dyspnea or a history of COVID-19 unprotected contact has to be tested for SARS-CoV2 infection and isolated until negative RT-PCR test. In addition to obtaining medical history, physical examination and laboratory tests, physicians may use different imaging techniques. Many studies of COVID-19 revealed CT scan abnormalities in both symptomatic and oligosymptomatic patients regardless of ICU stay, with typical CT features described as bilateral pulmonary parenchymal ground glass and peripheral consolidative pulmonary opacities (1-3). What more, Zhang et al. proposed including CT in the diagnostic strategy for COVID-19 in both febrile and afebrile lymphopenic patients, but routine CT screening is not practically implementable (2). While high resolution computer tomography (HRCT) is the gold standard for lung imaging in the diagnosis of diseases affecting the pulmonary interstitium, lung ultrasound (LUS) is being increasingly used. Diagnostic accuracy of a full LUS is $97 \%$ for consolidation and $95 \%$ for alveolar-interstitial syndrome and may be comparable to that of computed tomography (4). Up till now already numerous studies highlight the clinical value of LUS in COVID-19 diagnostic $(5,6)$.

Moreover, in COVID19 pneumonia, complete LUS may be of comparable value to CT both as a diagnostic and prognostic tool for the disease progression (7-10).

Recent studies suggest that LUS may be a useful tool to monitor SARS-CoV2 infection, assess the COVID-19 severity, and identify patients who might require Extracorporeal Membrane Oxygenation (ECMO) therapy with a specificity of $90 \%(11,12)$. Therefore, it is reasonable to presume that a full LUS can give the physician almost as many important details as CT, but is much more assessable $(10,13)$. We suggest that LUS protocols should be considered as an important part of COVID-19 diagnosis. Also, while negative antigen test or even single negative RT-PCR test for SARS-CoV2 should not be considered as conclusive, we strongly believe that in order to prevent

^ ORCID: 0000-0003-1796-7659. 
too early termination of safety regime LUS should be performed in every patient prior the decision of ending isolation $(14,15)$. With abnormal LUS images patient should be than retested and considered as COVID-19 until a subsequent negative RT-PCR tests confirms otherwise.

\section{LUS}

\section{Equipment}

Based on our experience gained in dealing with other viral pneumonias we assume, that all models and types of ultrasound devices are sufficiently accurate in order to perform a LUS. Considering the examination technique and the contamination risk, we would suggest using a convex, microconvex or sector probe in the adult population. Sector probe, although not ideal for LUS, meets the criteria of a small and universal bedside diagnostic tool of initial wholebody ultrasound examination. Also, for lean adults or the pediatric population, a linear probe may be more accurate.

Although LUS may be performed with the use of any type and model of ultrasound device, at the time of the current pandemic there is need for deep decontamination of medical equipment (the whole device, not only the probe). Moreover, the ultrasound device should fulfill two major criteria: firstly, it should be of a small enough size which allows one to place the whole device and the probe within plastic disposable covers which should be exchanged (if possible) after each patient assessment. Secondly, the device should be easy to handle, both during examination and decontamination. In our opinion, remote portable devices should be used if possible.

Before an examination, the ultrasound device settings should be optimized as follows: (I) all options for the minimization or augmentation of B lines should be turned off (e.g., harmonic imaging, cross-sectional imaging etc.) or the "lung" preset setting should be turned on; (II) gain should be set to minimum for securing artifacts with better contrast; (III) the focus should be set at the level of the pleural line, (IV) and the depth of assessment should usually be set at a level of $10-15 \mathrm{~cm}$.

\section{Examination}

According to 2020 Recommendations for LUS in Internal Medicine, it is recommended to perform a full LUS from the top fields to the base of the lungs in all anterior, lateral and posterior lung fields using the sliding technique (13).
The full assessment of lung aeration should concern six quadrants separately for each lung as presented in Figure 1. We recommend this technique, as it allows one to perform a more accurate examination in comparison with protocols assessing only a few given points in emergency medicine, such as the BLUE protocol (16). This approach is due to the fact that the characteristic imaging features of COVID-19 are usually multifocal and surrounded by the normal lung parenchyma, the so-called spared areas (17). As in the supine position only the anterior and postero-lateral parts are accessible especially in conscious and cooperating patients, we strongly suggest performing a LUS for the maximum assessable lung area, with the patient in a sitting position, hands placed on their knees, and standard surgical mask securely covering their face. However, when dealing with critically ill patients in the supine position, the anterior and posterolateral areas should be examined using the sliding technique. Lung sliding should be assessed with the transducer placed transversely to the ribs to avoid diagnostic errors that may occur, e.g., in subcutaneous emphysema. Only after identifying the ribs and pleura, the probe should be placed longitudinally to the ribs in order to assess the maximum lung surface.

\section{Ultrasound findings}

At the early stage of COVID-19 lung involvement, focal artifacts of the B-lines are usually observed (17). The presence of a B-line pattern (three or more B-line artifacts) prove that the pulmonary parenchyma is already involved (18); multifocal B-lines are accompanied by small subpleural consolidations while irregular, thickened pleural line is observed. Pulmonary involvement in COVID-19 is usually subpleural, which facilitates LUS assessment sensitivity (19). These abnormalities are usually found in the lower and middle lung fields, in the posterolateral area (20). In the more advanced stage of the disease, abnormalities may be found in all lung fields (21). As with disease progression the number of B-lines increases, the lines start to fuse producing interstitial syndromes and, subsequently, "white lungs" (Figure 1G). Finally, the lungs may become atelectatic with diminished lung sliding. In each quadrant, the worst picture obtained should be evaluated on a scale according as proposed by Castelao et al. (22): 0 points (A pattern) - a lines with preserved pleural sliding, non-thickened and regular pleural line (Figure $1 F)$; 1 point (B7 pattern) - more than 3 regular B lines with separated from each other by $7 \mathrm{~mm}$ (Figure $1 E$ ); 

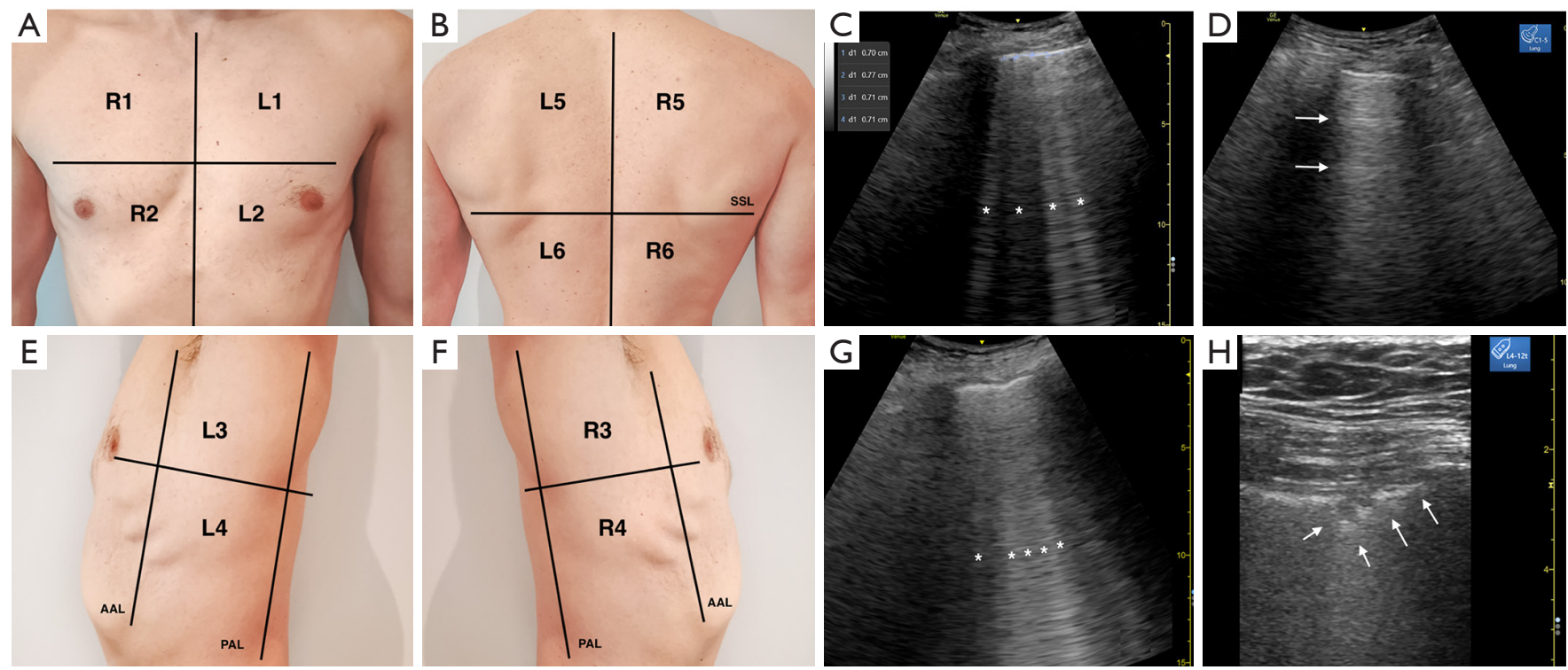

Figure 1 Division of each hemithorax into six quadrants limited (A) on the front by the sternal line and approximately fifth intercostal space (B); on the back by vertebral line and subscapular line (SSL); (C) on left and (D) right side of the thorax by anterior axillary line (AAL), posterior axillary line (PAL), and the line that runs in the middle of the distance between the diaphragm and the top of the axilla (approximately fifth intercostal space). The key artifacts observed during LUS are: clearly visible, sharp B-lines (asterix) appearing at 7 mm intervals, so called B7 profile (E); fully aerated lung areas with horizontal A-lines (arrow) representing pleural reverberations (during the examination pleural sliding was visible) (F); multiple merging B-line artifacts (asterix), so called B3 profile (G); local subpleural consolidations (arrow) (H).

2 points (B3 pattern) - more than $3 \mathrm{~B}$-lines separated from each other by $3 \mathrm{~mm}$, irregular or blurred (Figure 1G); 3 points (C pattern)—subpleural consolidations (Figure 1H). The obtained result ranges from 0 to 36 points. For better staff certainty we do recommend internal training organized by physician with best experience to avoid confusion caused by presence of irrelevant artifacts as I-lines and Z-lines (23).

\section{The protocol}

The protocol was designed primarily for patients treated in the emergency departments with conditions other than those considered infectious, such as: trauma; acute coronary syndrome; stroke; or other numerous indications for admission; and those requiring urgent surgical or medical treatment. Every patient with singular negative RT-PCR test and any of following: persistent flu-like symptoms (chills, cough, sore throat, myalgia, fatigue) or body temperature $\geq 37.3^{\circ} \mathrm{C}$, or dyspnoea (classified as NYHA II or increase at least of 1 NYHA point), or finally respiratory failure (classified as $\mathrm{SpO}_{2}<93 \%$ ), documented or suspected unprotected contact with COVID-19; should be evaluated with LUS before decision of terminating isolation despite negative antigen test or single RT-PCR negative test result (Figure 2). If the LUS reveals abnormalities suggesting viral pneumonia findings, the patient should be treated as a COVID-19 suspected case. In this case, although performing subsequent RT-PCR test should be considered as urgent, waiting for the test result should not delay proper, specific treatment. This procedure may prevent the uncontrolled spread of the virus and help to identify patients with mild symptoms, but significant lung involvement. Both the result of LUS equal to or greater than 19 and the score of 4 points on the anterior surface of the chest may suggest high risk of respiratory failure and the possible need for intensive care treatment.

\section{Future possibilities for assessment in the emergency medical services}

Patient prognosis in life-threatening situations is strongly dependent on accurate differential diagnosis and tailored treatment at all stages of care. Implementation of the safety protocol presented herein into prehospital patient care could potentially result in the implementation of adequate procedures at the site of the first intervention. 


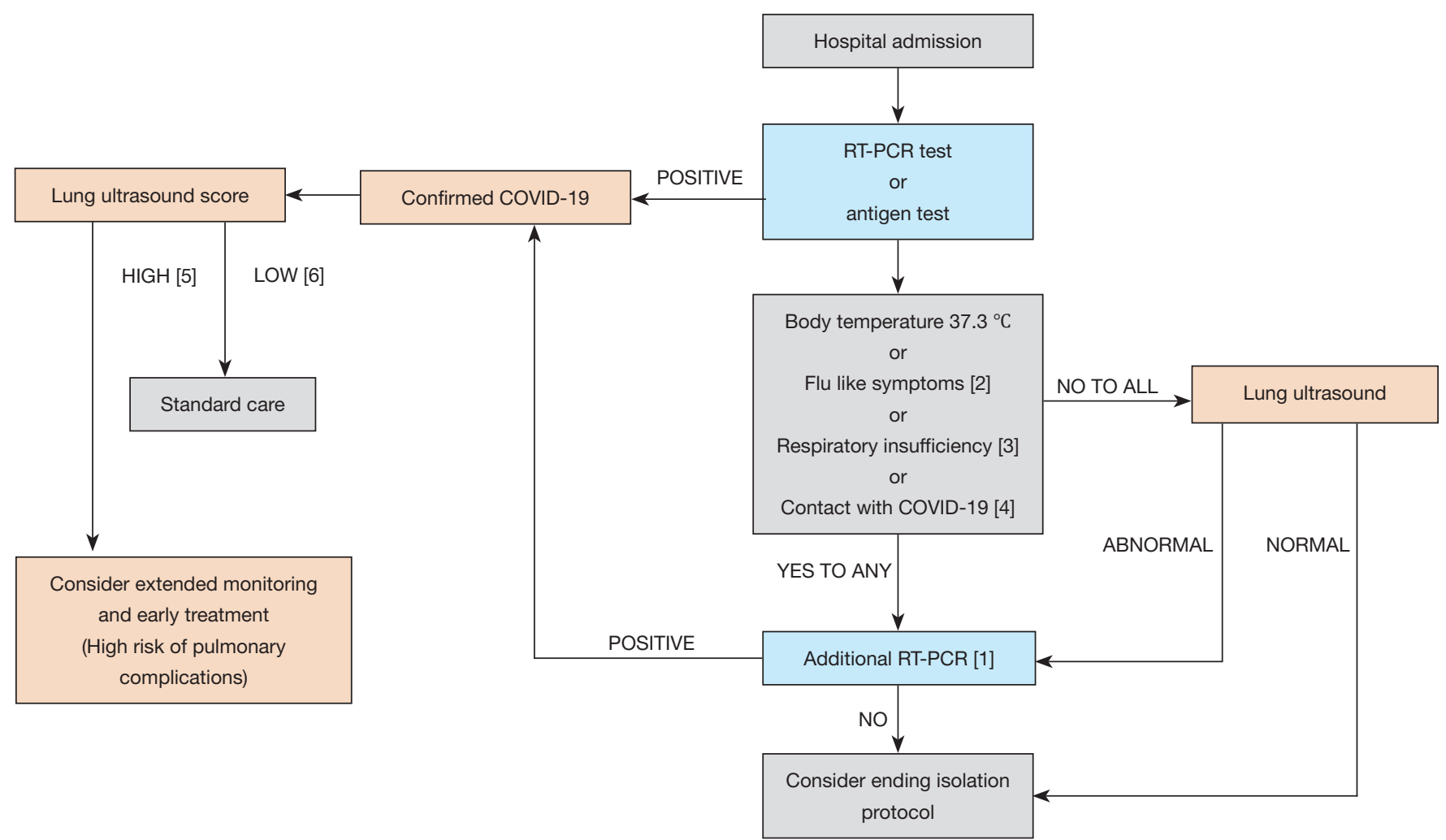

Figure 2 A proposed protocol for the lung ultrasound COVID-19 triage. [1] Preferably specimens collected from the lower respiratory tract; [2] persistent chills, cough, sore throat, myalgia, fatigue; [3] dyspnoea classified as NYHA II or increase at least of 1 HYHA point or $\mathrm{SpO}_{2}<93 \%$; [4] documented or suspected unprotected contact with COVID-19; [5] total LUS score $\geq 19$; or $\geq 4$ points on anterior chest wall; [6] total LUS of 0 makes pneumonia unlikely.

The aim of prehospital COVID-19 screening is to detect as many people as possible at high risk of severe COVID-19 infection. Onsite LUS assessment, enhanced by teletransmission, such as in the case of ECG, could augment patient diagnosis and facilitate better triage (transfer the subject directly to a hospital chosen to provide COVID-19 treatment). This pre-triage could potentially relieve emergency departments which deal with cases other than those involving COVID-19 patients.

\section{Discussion}

While RT-PCR is considered as a gold standard test to confirm SARS-CoV2 infection its reliability depends on several factors including sample quality, RNA stability, time of sample harvesting and laboratory errors. Also, the time is important, as the viral replication in upper respiratory tract is highest between $4^{\text {th }}$ and $10^{\text {th }}$ day since the onset of symptoms (24). Although bronchoalveolar lavage provide the most value material it causes increased exposition to aerosol potentially contaminated with SARS$\mathrm{CoV} 2$, requires significant health care resources, therefore nasopharyngeal swab is still the simplest, acceptable by patients and most commonly performed technique with sensitivity estimated at $63 \%(24,25)$. Fast antigen tests are significantly less sensitive than PCR testing, thus the high risk of false negative results of antigen tests and RT-PCR test emphasizes the need for implementing additional safety procedures.

Among different triage techniques, ultrasound chest imaging seems to be the most suitable in the current epidemiologic situation. Due to specific artifact analysis, both the risk of bias and inter-observer variability is negligible. Easy and quick training enables fast implementation of this technique into everyday practice, which can be crucial in reducing the risk of uncontrolled spread of COVID-19. There are no limitations to perform LUS, but there are specific conditions, in which the image may be ambiguous or even 
impossible to obtain, such as pneumothorax or excess of adipose tissue. We are also aware of the limited diagnostic capabilities of ultrasound and the risk of false positive results in geriatric population with numerous comorbidities and often coexisting heart failure. However, the proposed cutoff value of the LUS (19 points or 4 points on the anterior surface) seems to be reasonable. Moreover, the presence of subpleural consolidation may be of great diagnostic value for inflammatory changes. Additional echocardiographic evaluation should be performed whenever possible to exclude heart failure. We also want to emphasize that abnormal findings in LUS may last for up to several weeks, therefore this method may be of little use to the difference between ongoing inflammation and early post-COVID-19 changes. Nonetheless, LUS may be implemented at the early stage of patient evaluation in order to assess pulmonary causes of dyspnea and respiratory failure, as well as identify the patients with high risk of ARDS (22).

According to our best knowledge, recommendations proposed by the German Society of Ultrasound in Medicine (DEGUM) or British Medical Ultrasound Society (BMUS) concern mainly the imaging techniques and monitoring COVID-19 patients, but do not indicate the place of LUS in diagnostic algorithms $(26,27)$. Our protocol is one of the first attempts to facilitate hospital safety protocols in the current SARS-CoV2 pandemic. Patients transferred to the hospital may be asymptomatic or may have negative RT-PCR test result. Considering the unspecific signs and symptoms, patient assessment should be reinforced by an additional objective tool such as a LUS. The risk of admitting patient with false negative RT-PCR into unsecured setting may cause infection of numerous patients and employees or equipment contamination. We based our protocol on a need of both increase in-hospital patient safety and the need of monitoring severity of COVID-19 and early detection of patients who might require pharmacological treatment, mechanical ventilation or ECMO therapy. Current data suggest a median incubation period from 5 to 6 days (ranging, 1-14 days) and a high risk of asymptomatic carrier transmission during this period of time. According to the data from China, $1.2 \%$ of the population may be asymptomatic with as many as $80.9 \%$ patients presenting only mild upper respiratory tract symptoms and chills (28). It also has to be pointed out that pulmonary involvement in an oligosymptomatic or asymptomatic patient is frequently associated with a poorer prognosis (11).

Prior terminating isolation each patient with single negative RT-PCR or antigen test should be examined with a LUS and, when the examination proves to be abnormal, considered as SARS-CoV2 positive, until an additional RTPCR test result confirms otherwise. In this case, all personal protection equipment and hospital internal procedures should be implemented in order to maintain the safety of medical staff. In contrast, every patient with single negative RT-PCR test, fully aerated lungs and no previously discussed symptoms may be considered as at neglectable risk and treated with standard care. It is also important that the LUS score can be used to assess disease progression, indicating the need for CT scanning. Using LUS, it is possible to reduce the number of chest $\mathrm{X}$-rays and $\mathrm{CT}$ scans during this pandemic, which reduces radiological exposure, improves patient care efficiency and increases the availability of diagnostic tests (29). LUS is also a powerful tool in adjusting mechanical ventilation and can be used for noninvasive real-time monitoring of lung recruitment, which is a dynamic process. It allows detecting the actual opening and closing pressures of lungs, which in severe ARDS are an extremely heterogeneous organ. Thus, ultrasound can guide the way of an open-lung strategy, making it a goal-directed therapy (30). Finally, our assertion was partially confirmed by Volpicelli et al. who have shown that combining LUS with clinical presentation can be helpful in identifying patients with or without COVID-19 (31).

\section{Conclusions}

LUS seems to be one of the most assessable, easy to implement and resource saving tools, which may be practically included into safety protocols in order to improve patient and healthcare providers safety in the current COVID-19 pandemic. Both patients treated by the EMS and in-hospital patients should be evaluated with LUS for the risk of severe ARDS and possible complications.

\section{Acknowledgments}

Funding: None.

\section{Footnote}

Provenance and Peer Review: This article was commissioned by the editorial office, Fournal of Thoracic Disease. The article has undergone external peer review.

Conflicts of Interest: All authors have completed the ICMJE uniform disclosure form (available at http://dx.doi. 
org/10.21037/ jtd-21-295). The authors have no conflicts of interest to declare.

Ethical Statement: The authors are accountable for all aspects of the work in ensuring that questions related to the accuracy or integrity of any part of the work are appropriately investigated and resolved.

Open Access Statement: This is an Open Access article distributed in accordance with the Creative Commons Attribution-NonCommercial-NoDerivs 4.0 International License (CC BY-NC-ND 4.0), which permits the noncommercial replication and distribution of the article with the strict proviso that no changes or edits are made and the original work is properly cited (including links to both the formal publication through the relevant DOI and the license). See: https://creativecommons.org/licenses/by-nc-nd/4.0/.

\section{References}

1. Chung M, Bernheim A, Mei X, et al. CT Imaging Features of 2019 Novel Coronavirus (2019-nCoV). Radiology 2020;295:202-7.

2. Zhang J, Zhou L, Yang Y, et al. Therapeutic and triage strategies for 2019 novel coronavirus disease in fever clinics. Lancet Respir Med 2020;8:e11-2.

3. Ding X, Xu J, Zhou J, et al. Chest CT findings of COVID-19 pneumonia by duration of symptoms. Eur J Radiol 2020;127:109009.

4. Lichtenstein D, Goldstein I, Mourgeon E, et al. Comparative diagnostic performances of auscultation, chest radiography, and lung ultrasonography in acute respiratory distress syndrome. Anesthesiology 2004;100:9-15.

5. Smith MJ, Hayward SA, Innes SM, et al. Point-of-care lung ultrasound in patients with COVID-19 - a narrative review. Anaesthesia 2020;75:1096-104.

6. Buda N, Segura-Grau E, Cylwik J, et al. Lung ultrasound in the diagnosis of COVID-19 infection - A case series and review of the literature. Adv Med Sci 2020;65:378-85.

7. Global and Regional Diagnostic Accuracy of Lung Ultrasound Compared to CT in Patients With Acute Respiratory Distress Syndrome: Erratum. Crit Care Med 2020;48:e268.

8. Zieleskiewicz L, Markarian T, Lopez A, et al. AZUREA Network. Comparative study of lung ultrasound and chest computed tomography scan in the assessment of severity of confirmed COVID-19 pneumonia. Intensive Care Med 2020;46:1707-13.
9. Huang Y, Wang S, Liu Y, et al. A preliminary study on the ultrasonic manifestations of peripulmonary lesions of noncritical novel coronavirus pneumonia (COVID-19), 26 February 2020, PREPRINT (Version 1). Available online: https://doi.org/10.21203/rs.2.24369/v1

10. Peng QY, Wang XT, Zhang LN; Chinese Critical Care Ultrasound Study Group (CCUSG). Findings of lung ultrasonography of novel corona virus pneumonia during the 2019-2020 epidemic. Intensive Care Med 2020;46:849-50.

11. Zhao L, Yu K, Zhao Q, et al. Lung Ultrasound Score in Evaluating the Severity of Coronavirus Disease 2019 (COVID-19) Pneumonia. Ultrasound Med Biol 2020;46:2938-44.

12. Tung-Chen Y. Lung ultrasound in the monitoring of COVID-19 infection. Clin Med (Lond) 2020;20:e62-5.

13. Buda N, Kosiak W, Wełnicki M, et al. Recommendations for Lung Ultrasound in Internal Medicine. Diagnostics (Basel) 2020;10:597.

14. Di Paolo M, Iacovelli A, Olmati F, et al. False-negative RTPCR in SARS-CoV-2 disease: experience from an Italian COVID-19 unit. ERJ Open Res 2020;6:00324-2020.

15. Mendrala K, Skoczyński S, Darocha T, et al.: A SINGLE NEGATIVE SARS-COV2 RT-PCR TEST DOES NOT EXCLUDE COVID-19 IN PATIENT WITH SEVERE ARDS, CASE STUDY. Wiad Lek 2020;73:1800-3.

16. Bekgoz B, Kilicaslan I, Bildik F, et al. BLUE protocol ultrasonography in Emergency Department patients presenting with acute dyspnea. Am J Emerg Med 2019;37:2020-7.

17. Lu W, Zhang S, Chen B, et al. A Clinical Study of Noninvasive Assessment of Lung Lesions in Patients with Coronavirus Disease-19 (COVID-19) by Bedside Ultrasound. Ultraschall Med 2020;41:300-7.

18. Millington SJ, Koenig S, Mayo P, et al. Lung Ultrasound for Patients With Coronavirus Disease 2019 Pulmonary Disease. Chest 2021;159:205-11.

19. Nouvenne A, Zani MD, Milanese G, et al. Lung Ultrasound in COVID-19 Pneumonia: Correlations with Chest CT on Hospital admission. Respiration 2020;99:617-24.

20. Buonsenso D, Piano A, Raffaelli F, et al. Point-of-Care Lung Ultrasound findings in novel coronavirus disease-19 pnemoniae: a case report and potential applications during COVID-19 outbreak. Eur Rev Med Pharmacol Sci 2020;24:2776-80.

21. Soldati G, Smargiassi A, Inchingolo R, et al. Is There a Role for Lung Ultrasound During the COVID-19 Pandemic? J Ultrasound Med 2020;39:1459-62. 
22. Castelao J, Graziani D, Soriano JB, et al. Findings and Prognostic Value of Lung Ultrasound in COVID -19 Pneumonia. J Ultrasound Med 2020. [Epub ahead of print]. doi: 10.1002/jum.15508.

23. Lichtenstein DA. Lung ultrasound in the critically ill: the BLUE protocol. Cham: Springer, 2016.

24. CDC Interim Guidelines for Collecting, Handling, and Testing Clinical Specimens from Persons for Coronavirus Disease 2019 (COVID-19) 2020. Updated January 6, 2021, accessed February 17, 2021. Available online: https://www. cdc.gov/coronavirus/2019-ncov/lab/guidelines-clinicalspecimens.html

25. Wang X, Tan L, Wang X, et al. Comparison of nasopharyngeal and oropharyngeal swabs for SARSCoV-2 detection in 353 patients received tests with both specimens simultaneously. Int J Infect Dis 2020;94:107-9.

26. Kiefl D, Eisenmann S, Michels G, et al. German recommendations on lung and thoracic ultrasonography in patients with COVID-19. Med Klin Intensivmed Notfmed 2020;115:654-67.

27. The british medical ultrasound society COVID-19

Cite this article as: Skoczyński S, Buda N, Mendrala K, Górecki T, Kucewicz-Czech E, Krzych Ł, Koszutski T, Darocha T. Lung ultrasound may improve COVID-19 safety protocols. J Thorac Dis 2021;13(5):2698-2704. doi: 10.21037/jtd-21-295 resources. Lung Ultrasound Guidance. published 30th March 2020, accessed 17 january 2021. Available online: https://www.bmus.org/policies-statements-guidelines/ professional-guidance/covid-19-resources

28. China CDC Weekly, The Novel Coronavirus Pneumonia Emergency Response Epidemiology Team. The Epidemiological Characteristics of an Outbreak of 2019 Novel Coronavirus Diseases (COVID-19) - China, 2020. China CDC Weekly 2020;2:113-22.

29. Vetrugno L, et al. Our Italian experience using lung ultrasound for identification, grading and serial follow-up of severity of lung involvement for management of patients with COVID-19. Echocardiography 2020;37:625-7.

30. Tusman G, Acosta CM, Costantini M. Ultrasonography for the assessment of lung recruitment maneuvers. Crit Ultrasound J 2016;8:8.

31. Volpicelli G, Gargani L, Perlini S, et al. Lung ultrasound for the early diagnosis of COVID-19 pneumonia: an international multicenter study. Intensive Care Med 2021;47:444-54. 